\title{
Study on Fuel Utilization Dynamic model of a SOFC-GT Hybrid System Based on Deep Learning Technique
}

\author{
Jinwei Chen ${ }^{1, *}$, Yao Chen ${ }^{1}$, and Huisheng Zhang ${ }^{1}$ \\ ${ }^{1}$ The Key Laboratory of Power Machinery and Engineering of Education Ministry, Shanghai Jiao \\ Tong University, 200240 Shanghai, P. R. China
}

\begin{abstract}
In order to perform operation management tasks, including state monitoring and control strategy optimization, of a solid oxide fuel cell-gas turbine (SOFC-GT) hybrid system, a data-driven dynamic model based on deep learning technique of long short term memory (LSTM) network is developed to predict the behaviours of fuel utilization. In addition, a LSTM model with unsupervised deep auto-encoder (DAE) method was developed to extract the feature from input data. The comparison performance between the common LSTM model and DAELSTM model was investigated. The results show that the DAE-LSTM model can enhance the prediction performance. Moreover, the effect of data size was investigated. The results demonstrate that the unsupervised DAE-LSTM model trained by large data size can further improve the prediction performance. The maximum error is only 0.00529 , and average error decreases to 0.00025 . In conclusions, the unsupervised DAE-LSTM model is an effective approach to predict dynamic behaviours.
\end{abstract}

\section{Introduction}

Solid oxide fuel cell-gas turbine (SOFC-GT) hybrid system is one of the most promising solutions to the energy and environment issues due to high efficiency as well as ultra-low emissions [1-4]. However, SOFC-GT systems are highly nonlinear, multi-variable, and strongly coupled, causing highly complex thermodynamic performance [5]. An accurate model is an essential foundation of system the state monitoring, fault diagnosis, control strategy optimization.

Many researches have investigated the dynamic mechanism model of SOFC-GT systems from the first principles in order to analyse the dynamic behaviours in detail. Brouwer et al [6] developed a dynamic mechanism model for a SOFC-GT hybrid system with a $5 \mathrm{~kW}$ two shaft gas turbine. The dynamic performance with biomass gas was investigated for designing control system. Mclarty [7] developed a dynamic mechanism model with three-dimensional SOFC model to analysing the performance of a $100 \mathrm{MW}$ SOFC-GT system. Ferrari and Traverso et al $[8,9]$ developed TRANSEO program on the Simulink platform and established a dynamic mechanism model for a SOFC-GT system

* Corresponding author: chenjinweituihou@sjtu.edu.cn 
with anode ejector. These mechanism models describe the internal complex mass, heat and electrochemical processes, including the distribution of gas components, pressure, temperature, current density, voltage and other parameters. However, the mechanism model involves several physical properties and assumptions, such as the electronic and ionic conductivity, turbomachinery characteristics. The complexity and degradation of SOFC and gas turbine limit the comprehensiveness and accuracy of mechanism model.

The data-driven modeling method directly mines system characteristics from the input and output data. It has attracted huge attentions because of its efficiency and flexibility. Jurdo [10] established a nonlinear data-driven model with fuzzy Hammerstein method to predict the SOFC performance. Zhu et al [11-13] established several SOFC data-driven models based on various traditional machine learning algorithms such as neural network, support vector machine.

Benefitting from the prosperous development of big data analytics, deep learning technique is a promising approach to effectively mine the big data collected from operation and experiment [14]. The conventional machine learning methods transform the inputs with only one or two rounds before deriving the outputs, while the deep learning method transforms data with multiple times and automatically extract features from data with an unsupervised manner [15].

Fuel utilization is an important parameter to guarantee the SOFC operating effectively and safely. Therefore, the fuel utilization should be monitored and controlled at a proper range. In this paper, the supervised deep learning model and unsupervised deep learning model based on long short term memory (LSTM) network both developed to predict the dynamic performance of SOFC fuel utilization. The prediction performances of both LSTM models and the effect of training data size were also investigated.

\section{Methodology}

\subsection{System description}

The schematic of the SOFC-GT system fed by natural gas is shown in Fig. 1 which has been proposed in our previous work [16]. It is a pressurized hybrid system with anode and cathode recirculation loops, which are both performed by a single stage ejector. The fuel cell is a planar co-flow direct internal reforming SOFC.

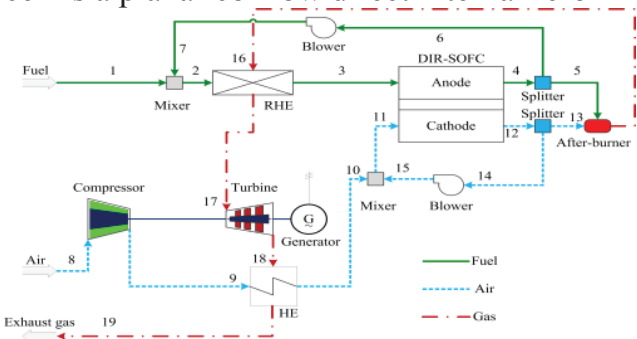

Fig. 1. Layout of the SOFC-GT system

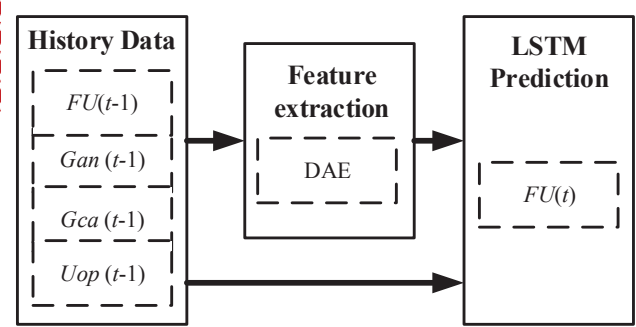

Fig.2. Structure of the DAE-LSTM model

\subsection{Dynamic LSTM model structure of fuel utilization}

The major factors affect the fuel utilization $(F U)$ are fuel flow rate $\left(G_{a n}\right)$, air flow rate $\left(G_{c a}\right)$, and SOFC operating voltage $\left(U_{o p}\right)$. Therefore, the LSTM model of fuel utilization is a multi-input single-output model. In additional, a deep auto-encoder (DAE) model is established to extract meaningful features as another input data of LSTM model. The structure of the DAE-LSTM model is shown in Fig. 2. 
In a physical SOFC-GT system, the LSTM training data can be sampled from the operating data or experiment data. However, with the limited availability of experiment and operation conditions, a mechanism model in our previous work [16, 17] is applied to supply the training data of LSTM model. The mechanism model includes SOFC model with four temperature layers, ejector model, compressor and turbine models based on characteristic map, lumped blower model and after-burner model, one dimensional heat exchanger (HE) model and reformer heat exchanger (RHE) model. The SOFC model is developed based on finite volume method and the detail SOFC modeling method and its validation can be seen in our previous work [17]. The ejector model simulate each section, including nozzle throat, nozzle outlet, mixing chamber inlet, diffusor inlet, and diffusor outlet. The detail modeling method can also be seen in our previous work [16].

\subsection{Feature extraction with DAE model}

Unsupervised deep learning has been widely applied to extract high-level abstract features from input data in several fields in order to reduce the risk of over-fitting [18]. The DAE algorithm is an unsupervised deep learning method, which consists of an encoder and a decoder. In this paper, a DAE model with a symmetric structure is established to extract the meaningful feature data, shown in Fig. 3.

The encoder maps the input data $\boldsymbol{x}$ to a hidden vector $\boldsymbol{h}$ through the sigmoid function.

$$
\boldsymbol{h}=s_{f}(\boldsymbol{W} \boldsymbol{x}+\boldsymbol{b})
$$

Where $s_{f}$ is the activation function of encoder, $\boldsymbol{W}$ and $\boldsymbol{b}$ are the parameters of encoder.

The decoder then transform the hidden vector $\boldsymbol{h}$ into a reconstruction vector $\mathbf{z}$.

$$
\mathbf{z}=s_{g}\left(\boldsymbol{W}^{\prime} \boldsymbol{h}+\boldsymbol{b}^{\prime}\right)
$$

Where $s_{g}$ is the activation function of decoder, $\boldsymbol{W}^{\prime}$ and $\boldsymbol{b}^{\prime}$ are the parameters of decoder.

The parameters of encoder and decoder $\boldsymbol{W}, \boldsymbol{b}, \boldsymbol{W}^{\prime}$, and $\boldsymbol{b}^{\prime}$ are optimized by minimizing the average reconstruction error.

$$
J_{A E}(\theta)=-\sum_{i=1}^{N}\left[x_{i} \log \left(z_{i}\right)+\left(1-x_{i}\right) \log \left(1-z_{i}\right)\right]
$$

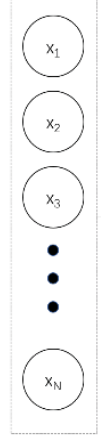

Fig. 3. Structure of DAE

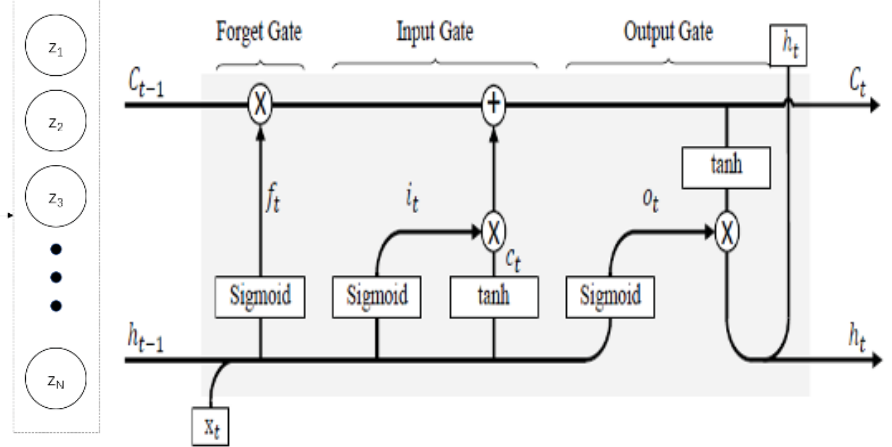

Fig. 4. Structure of LSTM

\subsection{LSTM prediction model}

LSTM is a special type of Recurrent Neural Network (RNN), which can effectively use lone-term data and solve the vanishing gradient problems of conventional RNN [19]. The structure of LSTM is shown in Fig. 4. Parameter C indicates the status of the cell and $\mathrm{h}$ indicates the output. There are three gates in LSTM, the forget gate determines which information of the previous steps should be discarded, the input gate determines which 
values of the input should be selected to update the state of the cell, the output gate then combines treated historical information with input data and achieve the final output [20].

\section{Result and discussion}

The capabilities of both LSTM model and DAE-LSTM model are tesed in a $328 \mathrm{~kW}$ SOFC-GT system shown in Fig. 1. The output powers of SOFC and GT are $270 \mathrm{~kW}$ and 58 $\mathrm{kW}$ resperistively, and the system efficiency can reach $61.88 \%$ at the design conditon. The detail thermodynamic performance can be seen in our previous work [16].

In order to compare their prediction performance of the common LSTM model and DAE-LSTM model, the network structures and parameters of activation function are optimized to established the optimal DAE and LSTM models. The DAE sturcture is composed of a 2-layer encoder with 4 and 2 nodes respectively. The structure of the decoder is symmetrical. The common LSTM network structure consists 15 nodes in its hidden layer and DAE-LSTM network sturcture is composed of 20 nodes. The batch size of the DAE and LSTM are both 200.

\subsection{Comparison of LSTM model and DAE-LSTM model}

After choosing the network parameters of LSTM model and DAE-LSTM model, the training data are sampled from the mechanism model when the fuel flow rate steps from $0.007 \mathrm{~kg} / \mathrm{s}$ to $0.0109 \mathrm{~kg} / \mathrm{s}$, and the size of sample data is 1000 . The test data are also sampled when the fuel flow rate steps from $0.007 \mathrm{~kg} / \mathrm{s}$ to $0.0095 \mathrm{~kg} / \mathrm{s}$.

Fig. 5 shows the comparison result of fuel utilization prediction performance between LSTM model and DAE-LSTM model. The maximum errors of LSTM and DAE-LSTM model both occur at the moment of step. The maximum error of common LSTM model is 0.00702 , and the DAE-LSTM model is 0.00690 . The average errors of common LSTM model and DAE-LSTM model are 0.00198 and 0.00102 , respectively. Therefore, the deep learning model using an unsupervised manner to extract feature as another input data can enhance the prediction performance.

Table 1. Errors between DAE-LSTM and LSTM

\begin{tabular}{|c|c|c|}
\hline & DAE-LSTM & LSTM \\
\hline Average error & 0.00102 & 0.00198 \\
\hline Maximum error & 0.00690 & 0.00702 \\
\hline
\end{tabular}



Fig. 5. Effect of unsupervised DAE

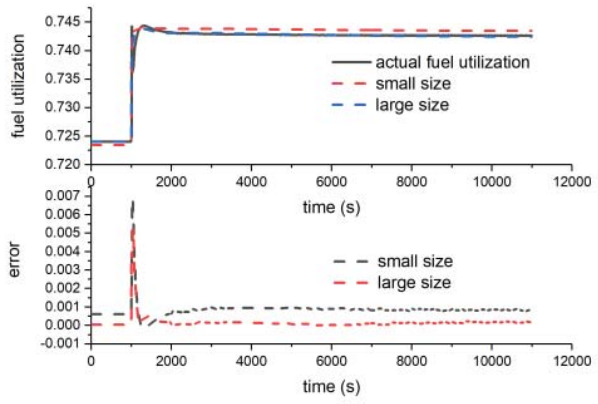

Fig. 6. Effect of training data size 


\subsection{Effect of training data size}

The accuracy of data-driven model increases with the growth of training data size because more useful information is included. Considering the prediction performance of common LSTM model and DAE-LSTM model, the DAE-LSTM model is applied to analyze the effects of training data size. Except the training data sampled in 3.1 section, a large training data are sampled with four steps of fuel flow rate, which steps from $0.007 \mathrm{~kg} / \mathrm{s}$ to $0.00895 \mathrm{~kg} / \mathrm{s}$, from $0.00895 \mathrm{~kg} / \mathrm{s}$ to $0.0109 \mathrm{~kg} / \mathrm{s}$, from $0.0109 \mathrm{~kg} / \mathrm{s}$ to $0.00895 \mathrm{~kg} / \mathrm{s}$, and from $0.00895 \mathrm{~kg} / \mathrm{s}$ to $0.007 \mathrm{~kg} / \mathrm{s}$ respectively. The size of large data is 4000 .

Fig. 6 demonstrates the effect of the data size on the prediction performance of DAELSTM model. The errors between small size data and large data size are shown in Table 2. The maximum errors of DAE-LSTM model trained by large size data is 0.00529 , and the average errors is only 0.00025 . Therefore, the prediction performance of DAE-LSTM model trained by large size data is more accurate than the model trained by small size data. In conclusion, the DAE-LSTM model with large size data is accurate enough to predict the dynamic performance of SOFC fuel utilization.

Table 2. Error between large size and small size

\begin{tabular}{|c|c|c|}
\hline & Large size & Small size \\
\hline Average error & 0.00025 & 0.00102 \\
\hline Maximum error & 0.00529 & 0.00690 \\
\hline
\end{tabular}

\section{Conclusions}

In this paper, a DAE-LSTM model is established to describe the fuel utilization dynamic behaviors of a SOFC-GT hybrid system. The conclusions are as following.

(1) A DAE-LSTM model and common LSTM model are developed to predict the SOFC fuel utilization dynamic behaviors. The unsupervised DAE model is designed to extract meaningful features from data. The LSTM models are developed to predict the fuel utilization performance.

(2) The LSTM model with DAE can enhance the prediction performance because the unsupervised manner DAE method can extract feature as another input data When the DAE model is added to extract features, the average error of prediction performance decreases from 0.00198 to 0.00102 . The maximum error decreases from 0.00702 to 0.00690 .

(3) The accuracy of the DAE-LSTM model increases with the growth of training data size. The average error with large size data decreases to 0.00025 , and maximum error decreases to 0.00529 .

\section{Acknowledgements}

This work was Funded by Program of China Postdoctoral Science Founation (Grant No. 2019M650085), National Fundenmental Research Project (Grant No. JCKY2017208A001) and National Natural Science Foundation of China (Grant No. 51876116).

\section{References}

1. N. Harun, D. Tucker, T. Adams, Open Loop and Closed Loop Performance of Solid Oxide Fuel Cell Turbine Hybrid Systems During Fuel Composition Changes, J. Eng. Gas. Turbines Power, 139(6), 061702, (2017). 
2. L. Larosa, M. L. Ferrari, M. Loredana, A. Massardo, SOFC/MGT Coupling: Different Options With Standard Boosters, ASME Paper GT2013-94072, (2013).

3. T. Choudhary, Thermodynamic assessment of advanced SOFC-blade cooled gas turbine hybrid cycle, Int. J. Hydrog. Energy, 42(15), 10248-10263, (2017).

4. M. L. Ferrari, E. Liese, D. Tucker, L. Lawson, A. Traverso, A. Massardo, Transient modeling of the NETL hybrid fuel cell/gas turbine facility and experimental validation, J. Eng. Gas. Turbines Power, 129(4), 1012-1019, (2007).

5. J. Chen, Y. Chen, H. Zhang, S. Weng, Effect of different operating strategies for a SOFC-GT hybrid system equipped with anode and cathode ejectors, Energy, 163, 1-14, (2018).

6. T. Kaneko, J. Brouwer, G. Samuelsen, Power and temperature control of fluctuating biomass gas fueled solid oxide fuel cell and micro gas turbine hybrid system, J. Power Sources, 160(1), 316-325, (2016).

7. D. Mclarty, J. Brouwer, S. Samuelsen, Fuel cell-gas turbine hybrid system design part II: Dynamics and control, J. Power Sources, 254(15), 126-136, (2014).

8. M. L. Ferrari, A. Traverso, A. Massardo, Transient analysis of solid oxide fuel cell hybrids: part B-Anode Recirculation Model, ASME Paper GT2004-53716, (2004).

9. L. Magistri, M. L. Ferrari, A. Traverso, P. Costamagna, A. Massardo, Transient Analysis of Solid Oxide Fuel Cell Hybrids: Part C-Whole-Cycle Model, ASME Paper GT2004-53845, (2004).

10. F. Jurado, Modeling SOFC plants on the distribution system using identification algorithms, J. Power Sources, 129(2), 205-215, (2004).

11. X. Wu, X. Zhu, G. Cao, H. Tu, Predictive control of SOFC based on a GA-RBF neural network model, J. Power Sources, 179(1), 232-239, (2008).

12. H. Huo, Z. Zhong, X. Zhu, H. Tu, Nonlinear dynamic modeling for a SOFC stack by using a Hammerstein model, J. Power Sources, 175(1), 441-446, (2008).

13. X. Wu, X. Zhu, G. Cao, H. Tu, Nonlinear modeling of a SOFC stack based on ANFIS identification, Simul. Model. Pract. Theory, 16(4), 399-409, (2008).

14. J. Schmidhuber, Deep learning in neural networks: an overview, J. Neural Netw, 61, 85-117, (2015).

15. Y. Lecun, Y. Bengio, G. Hinton, Deep learning, Nature, 521(7553), 436-444, (2015).

16. J. Chen, K. Gao, M. Liang, H. Zhang, Performace Evaluation of a SOFC-GT Hybrid System with Ejectors for the Anode and Cathode Recirculations, J. Electrochem. Energy Convers. Storage, 16(4), 041004, (2019).

17. J. Chen, H. Zhang, S. Weng, Study on Nonlinear Identification SOFC Temperature Model Based on Particle Swarm Optimization-Least Squares Support Vector Regression, J J. Electrochem. Energy Convers. Storage, 14(3), 031003, (2017).

18. T. Chen, C. Guestrin, Xgboost: A scalable tree boosting system, ACM conference on knowledge discovery and data mining, 785-794, (2017).

19. S. Hochreiter, J. Schmidhuber, Long Short-term Memory, Neural Computation, 9(8), 1735-1780, (1997).

20. J. James, A. Lam, D. Hill, V. Li. Delay Aware Intelligent Transient Stability Assessment System, IEEE Access, 5, 17230-17239, (2017). 